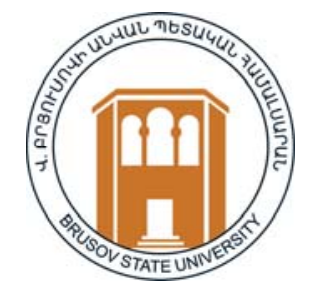

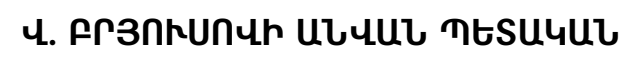
CUUULUURUL

ГОСУДАРСТВЕННЫЙ УНИВЕРСИТЕТ

ИМЕНИ В. БРЮСОВА

BRUSOV STATE UNIVERSITY

คulคtr

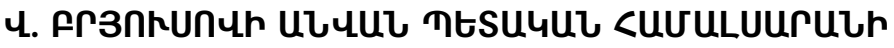
ВЕСТНИК ГОСУДАРСТВЕННОГО УНИВЕРСИТЕТА ИМЕНИ

B. БРЮСОВА

BULLETIN OF BRUSOV STATE UNIVERSITY

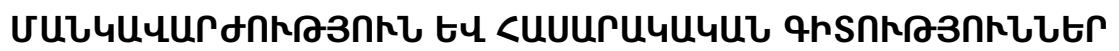

ПЕДАГОГИКА И СОЦИАЛЬНЫЕ НАУКИ

PEDAGOGY AND SOCIAL SCIENCES

2(55)

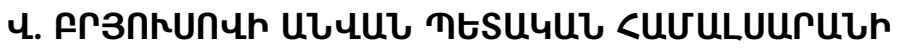

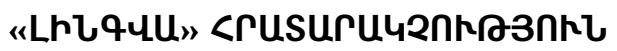

เГヒบuし - 2021 
CS? 378.147:811.111

DOI: 10.51307/182931072015233260/21.2-97

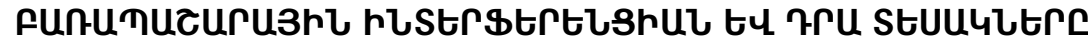

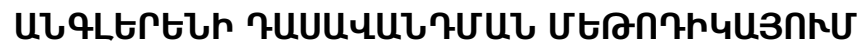

\section{<UUUnц Anzurзul}

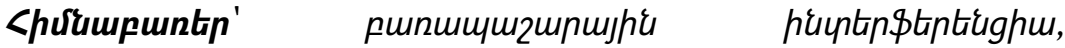

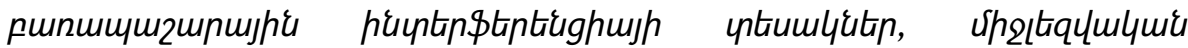

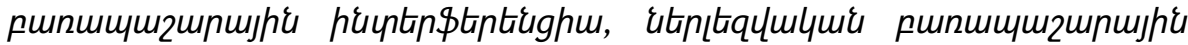 \\ husntiplantiughum
}

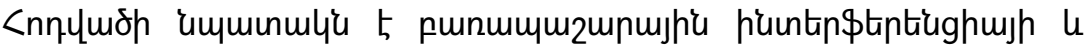

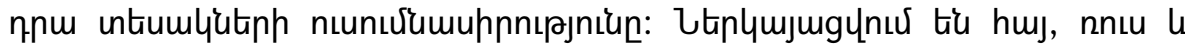

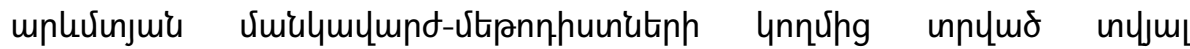

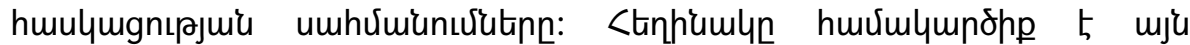

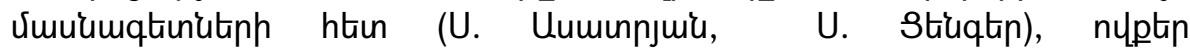

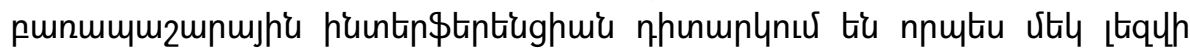

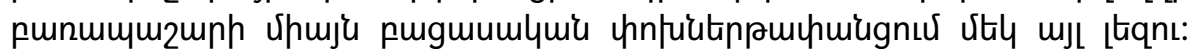

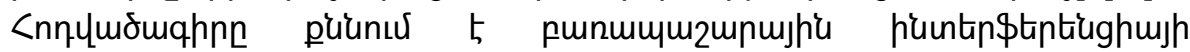

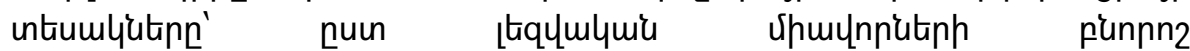

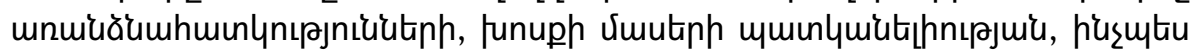

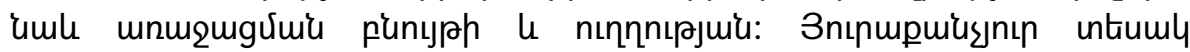

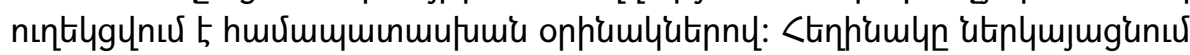

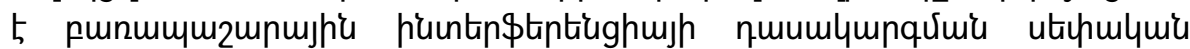

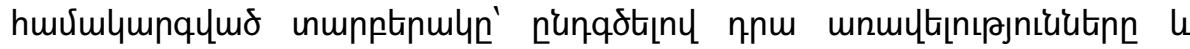

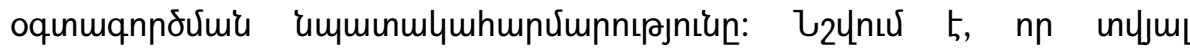

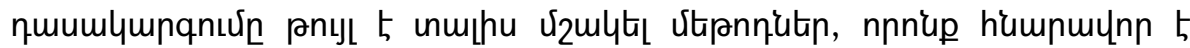

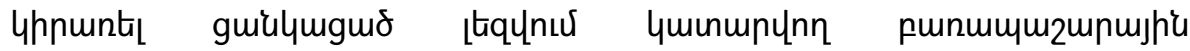

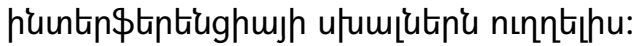

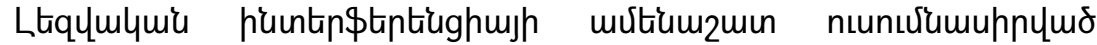

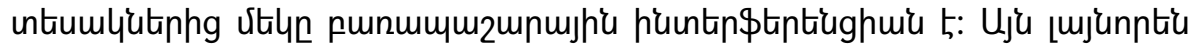

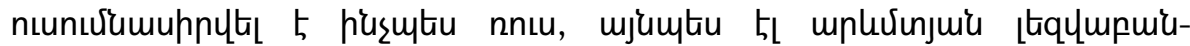

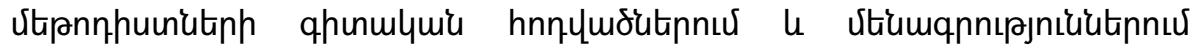
(Алимов 2005: 153, Шимановская 2014: 390, Ценгер 2014: 20, Dweik \& Othman 2017: 67, Kushnaryova 2016: 187): 


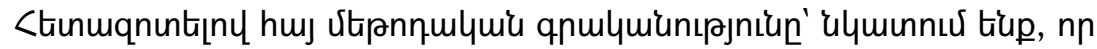

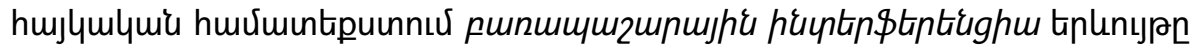

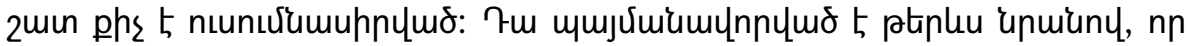

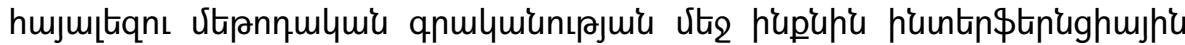

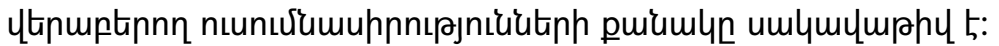

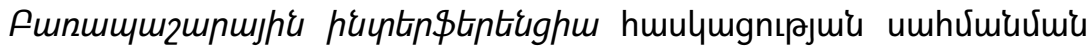

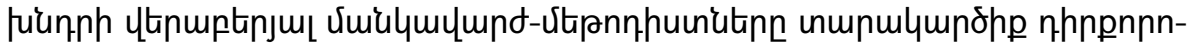

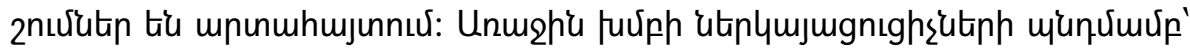

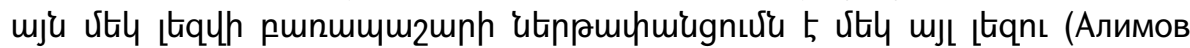

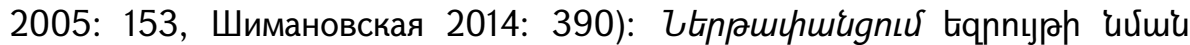

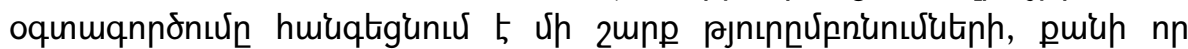

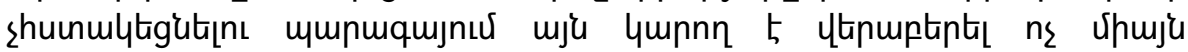

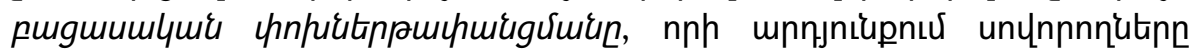

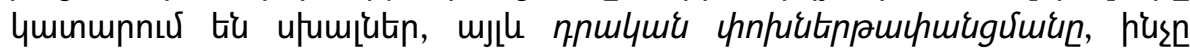

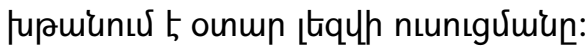

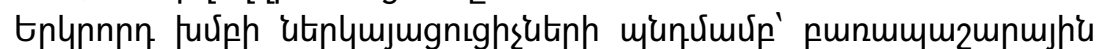

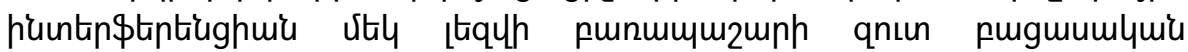

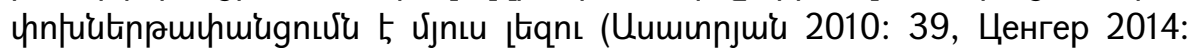

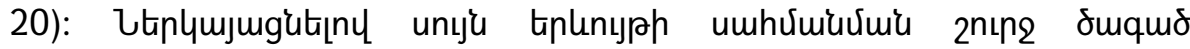

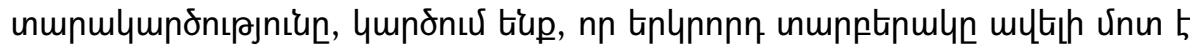

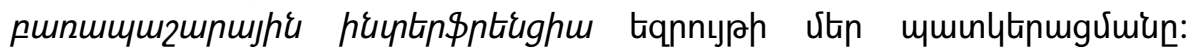

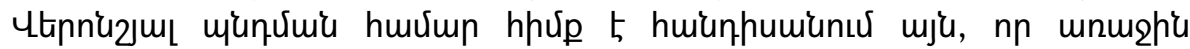

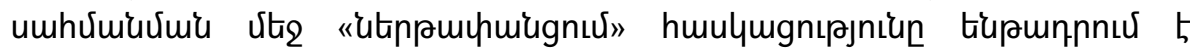

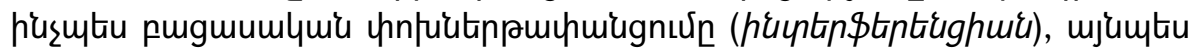

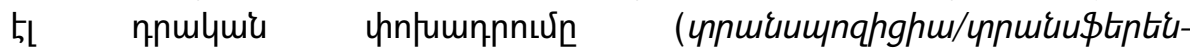

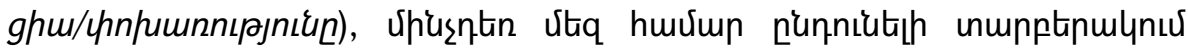

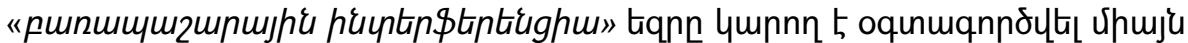

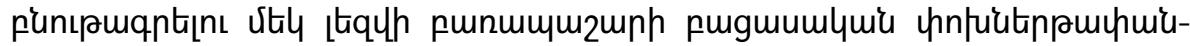
gnuर्u ujnıu jtqnı:

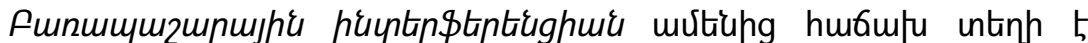

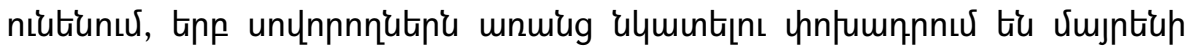

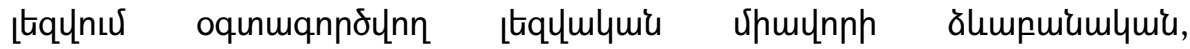

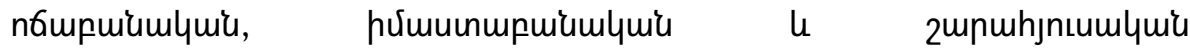

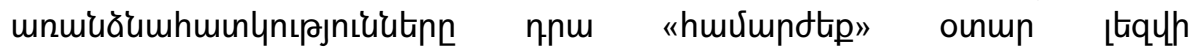

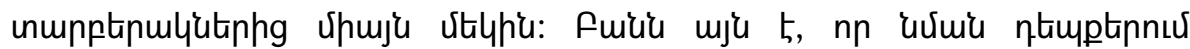

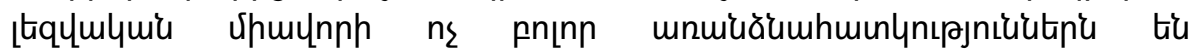

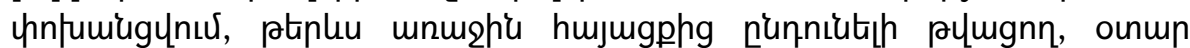

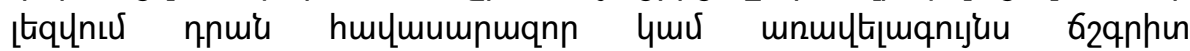

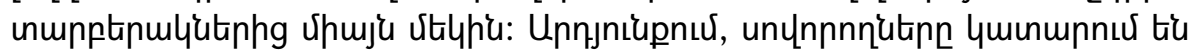




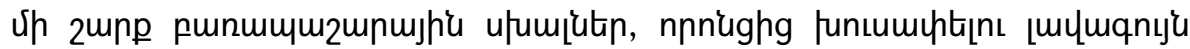

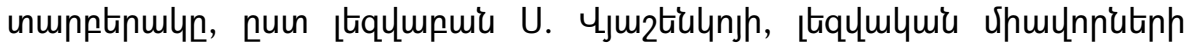

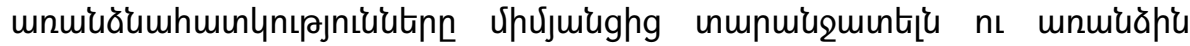

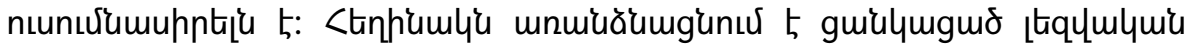

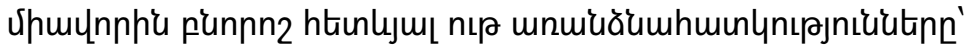

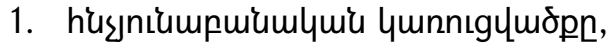

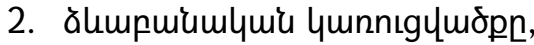

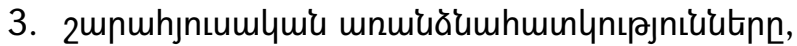

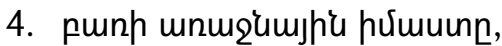

5. punh ınugnighs houmuinn,

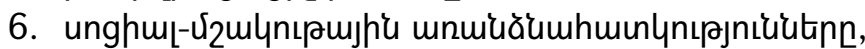

7. undnıрujunıрjnı́un,

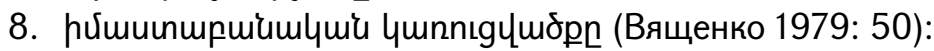

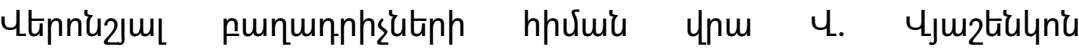

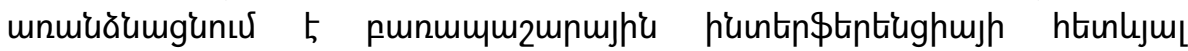
unhultnn'

1. Cus

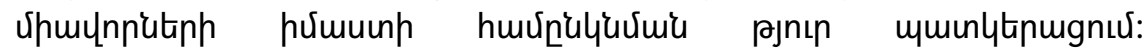

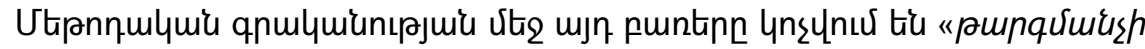

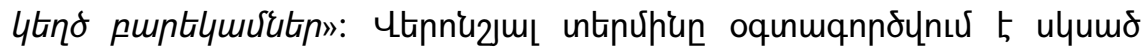

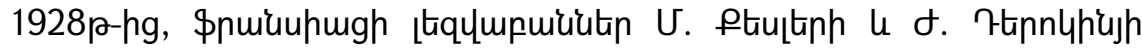

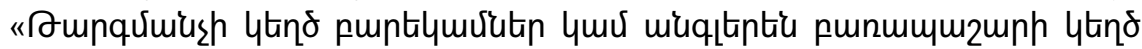

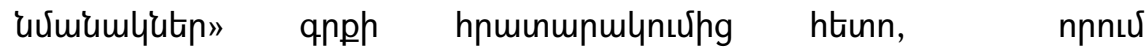

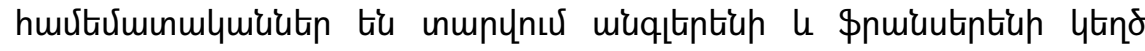

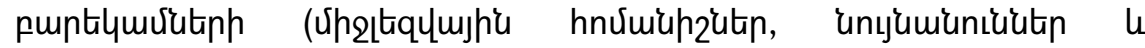
hupuiuniuutin) unglu (Koessler et Deroquigny 1928: XI): Tpununlytúp пnu up puiuh ophimulutin:

\begin{tabular}{|c|c|c|}
\hline UqqLtptu & <ujtptic & nnuutptis \\
\hline 1. Dutch & 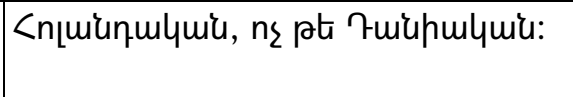 & $\begin{array}{l}\text { Голландский, ns pt } \\
\text { датский. }\end{array}$ \\
\hline 2. Physician & foh24, ptrnumulun, ns pt \$hqhynu: & Врач, n乏 pt фризик. \\
\hline 3. Magazine & Uরumqhn, n乏 pt fumiunıp: & $\begin{array}{l}\text { Журнал, ns } \\
\text { магазин. }\end{array}$ \\
\hline
\end{tabular}

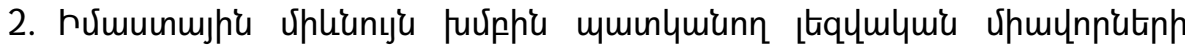

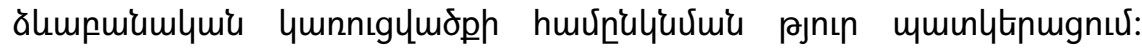
Onhimul'

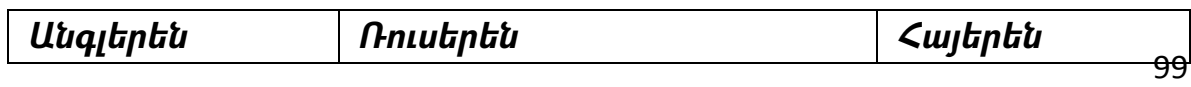




\begin{tabular}{|l|l|l|}
\hline $\begin{array}{l}\text { 1. Composer, n\& pt } \\
\text { compositor. }\end{array}$ & Композитор & 4nưumqhunnn \\
\hline $\begin{array}{l}\text { 2. Architect, n\& pt } \\
\text { architector. }\end{array}$ & Архитектор & бupunumumutin \\
\hline
\end{tabular}

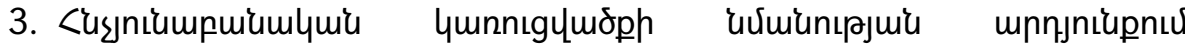

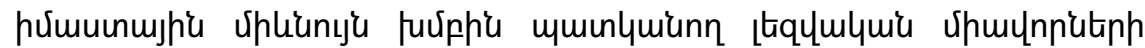

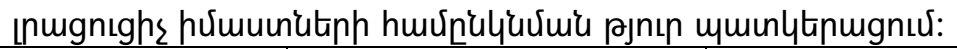

\begin{tabular}{|c|c|c|}
\hline Uuqutptú & Nncutptu & curjtintis \\
\hline 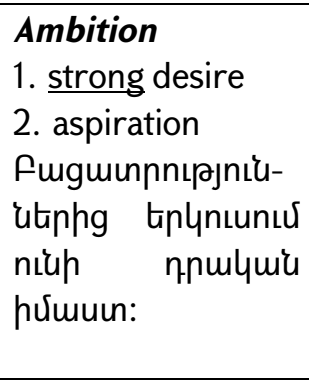 & 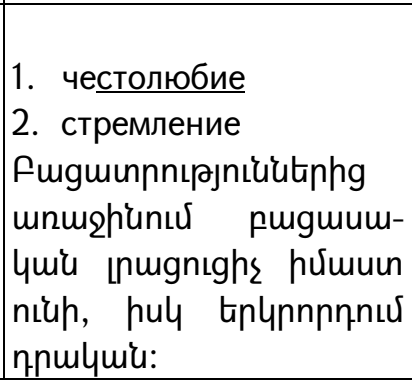 & 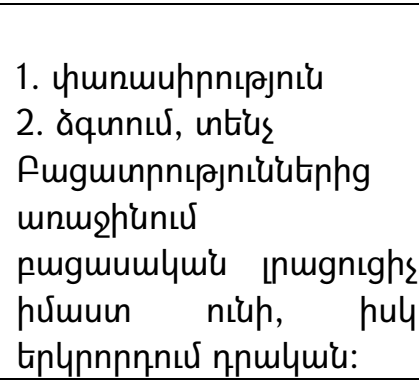 \\
\hline
\end{tabular}

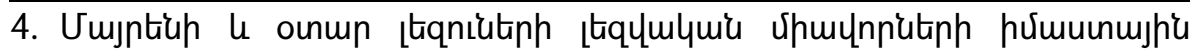

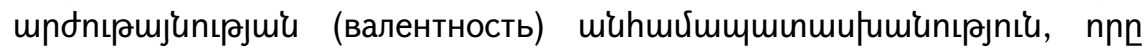

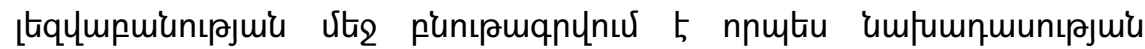

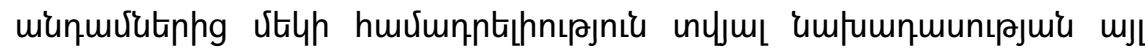

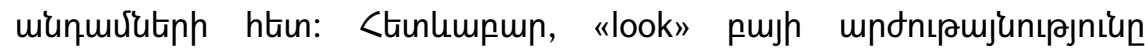

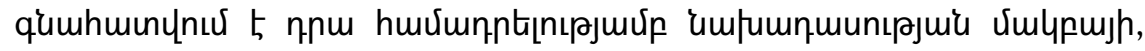

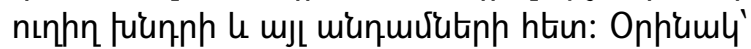

\begin{tabular}{|c|c|c|}
\hline Cunjtitiu & $\begin{array}{l}\text { Urqutiptí } \\
\text { unupptipuly }\end{array}$ & $\begin{array}{l}\text { Cujtintiup } \\
\text { huuntp\$tptinghujh } \\
\text { upnjniup }\end{array}$ \\
\hline U2humphn 4 inn 5 & It's a small world & It's a round world \\
\hline 7mирuі4 huјшgp & Blank look & Empty look \\
\hline 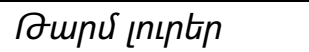 & Latest news & Fresh news \\
\hline
\end{tabular}

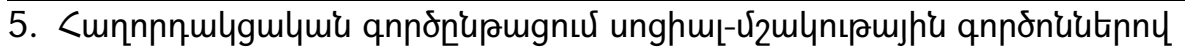

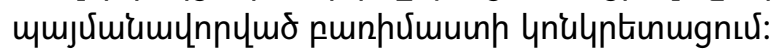

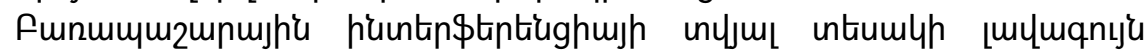

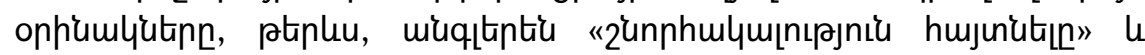

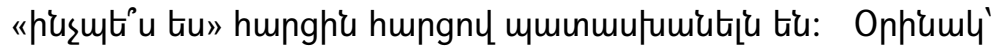




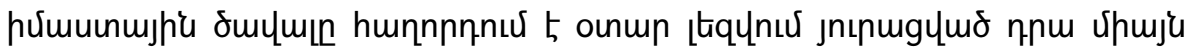
vity ununptipulyhu (Вященко 1979: 26):

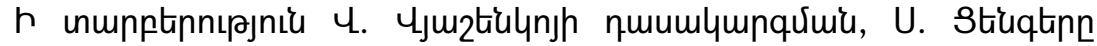

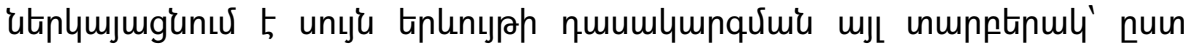

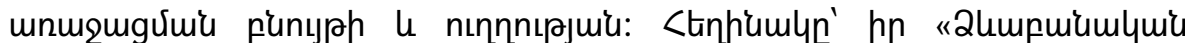

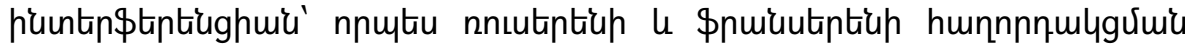

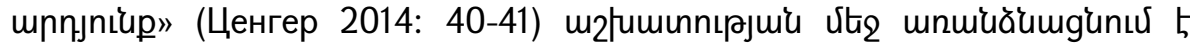

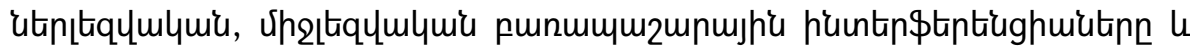
pulyunnıpjniun:

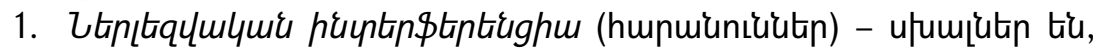

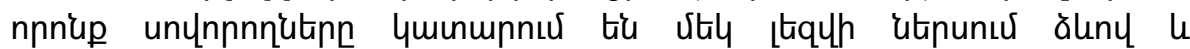

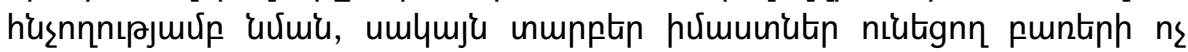

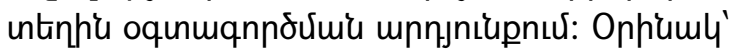

- estimate - estimation - esteem

- effective - efficient

- sensitive - sensible

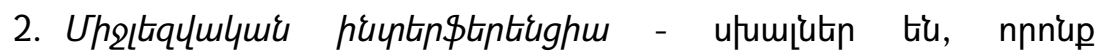

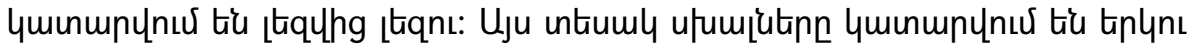

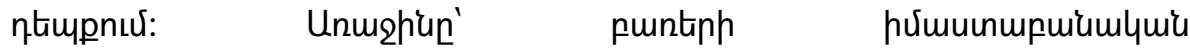

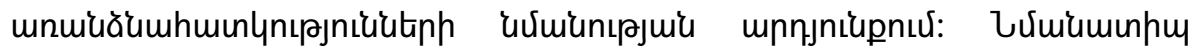

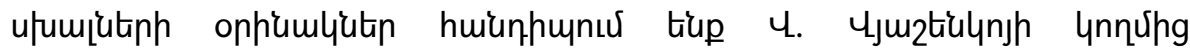

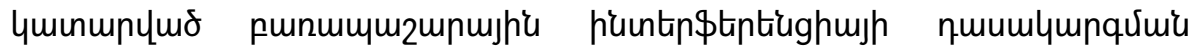
ununptinulyutrnnu:

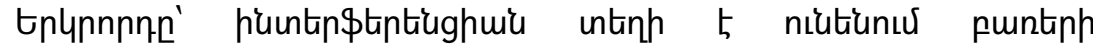

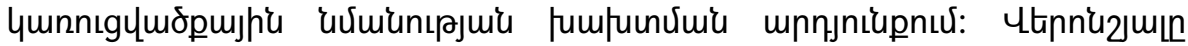

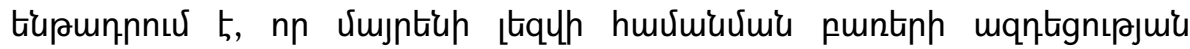

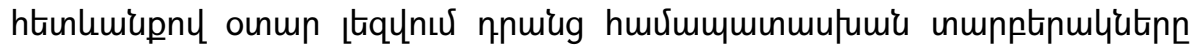

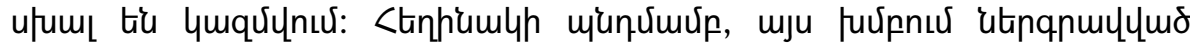

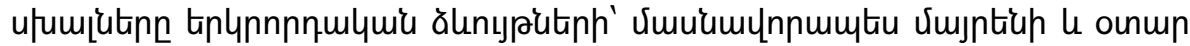

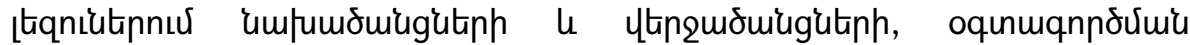

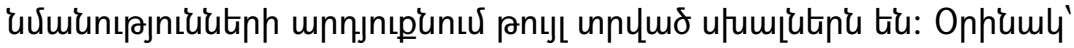

Un-, ir-, non- im-, in-, a-, dis-, mis-, ab- duunulyuiu hưuuun

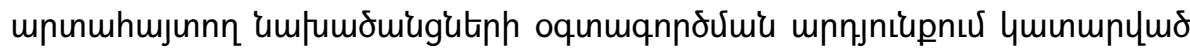
ułumןtuinn:

\begin{tabular}{|c|c|c|}
\hline 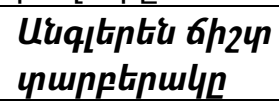 & $\begin{array}{l}\text { Uiqultptis upuml } \\
\text { nupptpuly }\end{array}$ & $\begin{array}{l}\text { Sujthntis } \\
\text { yumptrulyn }\end{array}$ \\
\hline Amoral & Inmoral, immoral, unmoral & 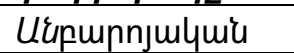 \\
\hline Abnormal & Unnormal, innormal & Uนiunnưul \\
\hline Impolite & Inpolite, unpolite & Uदрuпшршцшрh \\
\hline
\end{tabular}


Ultra-, super-, hyper-, over- punupưuunh hưuuunn nünupămlynn

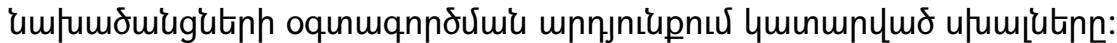

\begin{tabular}{|c|c|c|}
\hline $\begin{array}{l}\text { Uuquthtis 6h2un } \\
\text { unupptinuln }\end{array}$ & 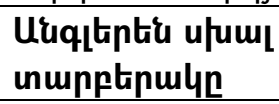 & Cujtptí unupptinulyn \\
\hline Overestimate & Superestimate & 9tpmuqumbuintil \\
\hline Ultramodern & Supermodern & qtinunnuălu \\
\hline Hyperactive & Superactive & 9tpnulyunhy \\
\hline
\end{tabular}

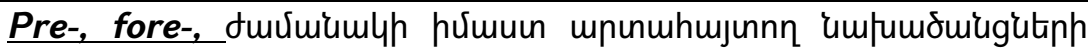

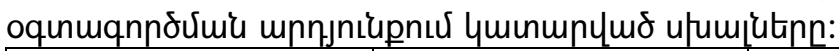

\begin{tabular}{|c|c|c|}
\hline $\begin{array}{l}\text { Uuq1tentis 6h2un } \\
\text { numptpuly }\end{array}$ & 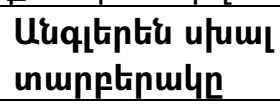 & Cujtentú ununptrulyn \\
\hline Foretell & Pretell & Zmpumqnızmutal \\
\hline Forefather & Prefather & Zm/uwhump \\
\hline Predestine & Foredestine & Zmphmumhưuital \\
\hline
\end{tabular}

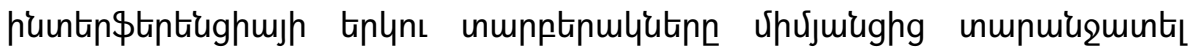

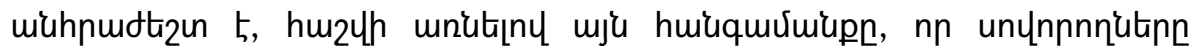

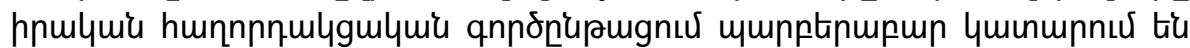

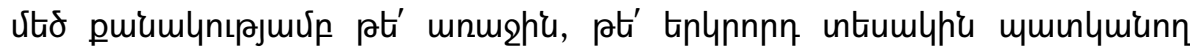
ułumgutin:

3. คuцunnцpјnıध (паронимическая аттракция) - ułumןutin tiu,

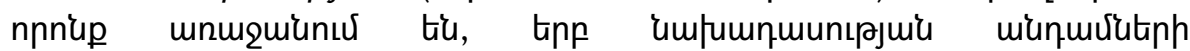

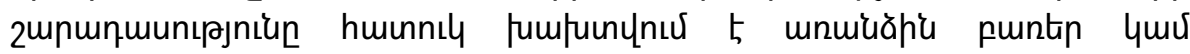

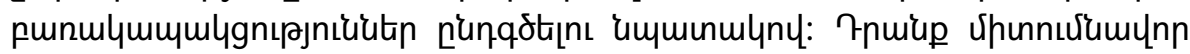

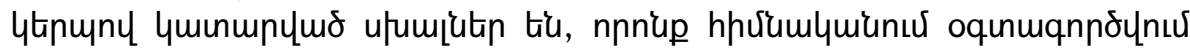

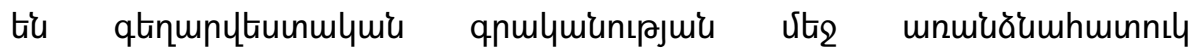

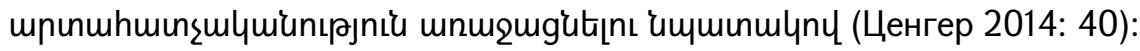

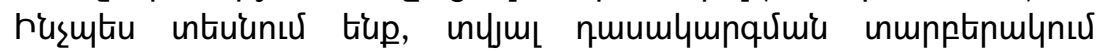

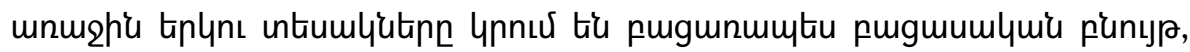

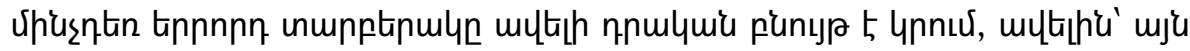
upume sh

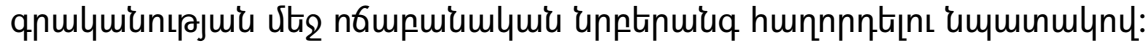

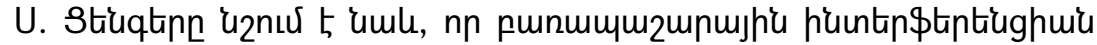

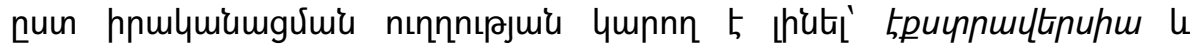

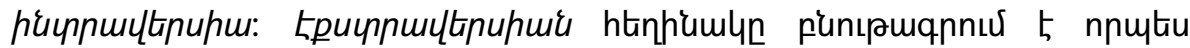

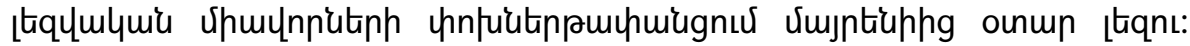

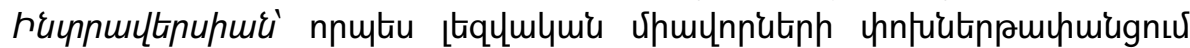
ounun jtqulhg Uumptiup ןtaqn (Ценгер 2014: 40): 


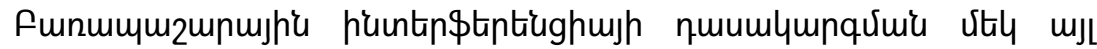

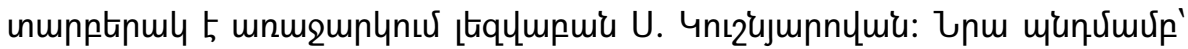

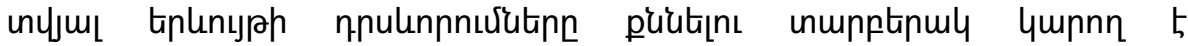

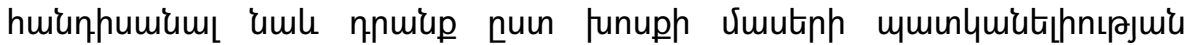

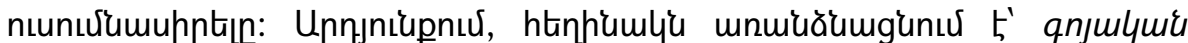

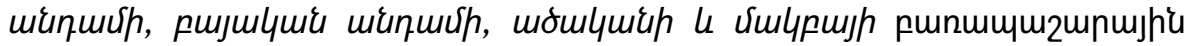
hiuntip\$tiptiughujp untumlyutinn (Kushnaryova 2016: 188):

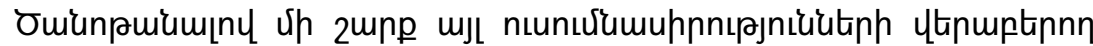

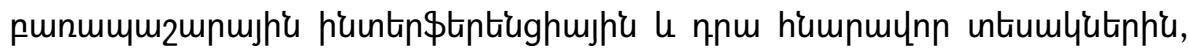

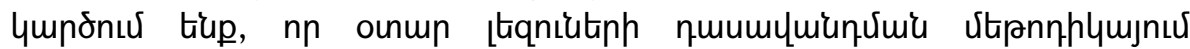

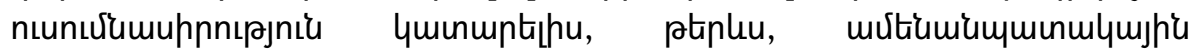

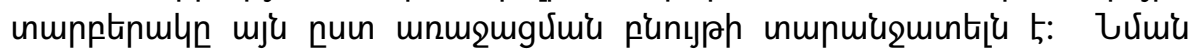

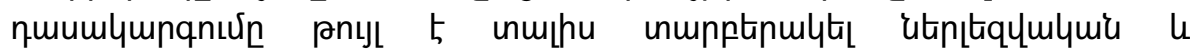

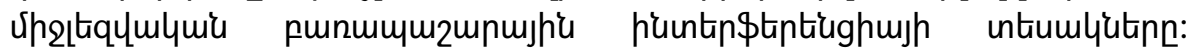

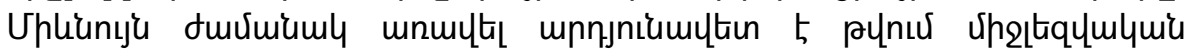

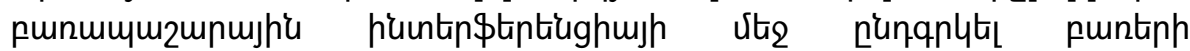

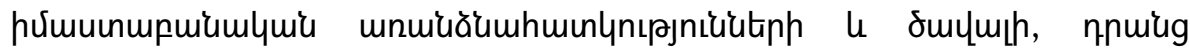

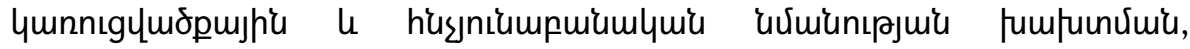

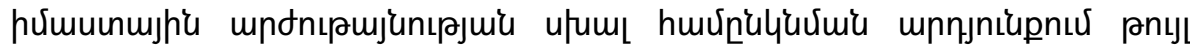

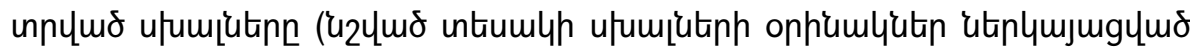

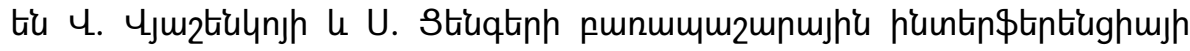

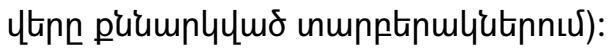

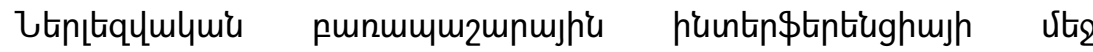

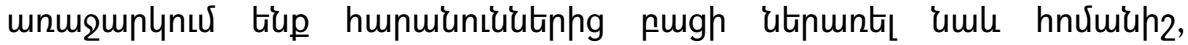

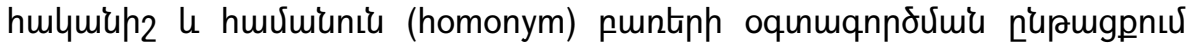
mpцmo uүumןutinn:

1. ¿numunh2 puntiph fuরuptin'

- show -display - exhibit - present - point to - indicate - demonstrate

-wrong - incorrect - inaccurate - mistaken - improper - erroneous

- fain - just - impartial - unbiased - objective - unprejudiced - honest

2. ¿mlumun2 puntinh fuরintin'

- arrive - leave - depart - go

- tough - soft - fragile - easy

- expand - contract - condense - shrink

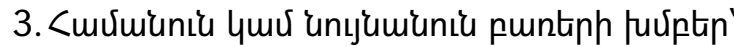

- for - four - fore

- pair - pear - pare

-there - their - they're 


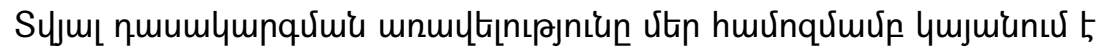

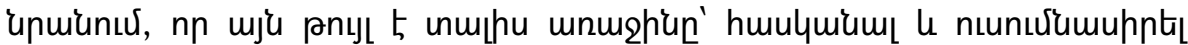

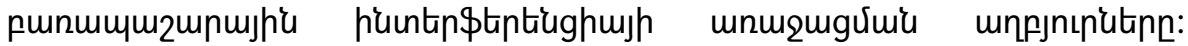

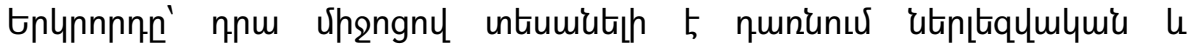

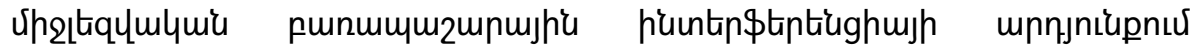

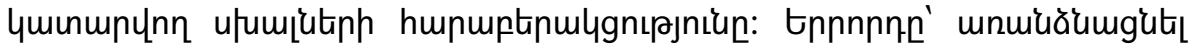

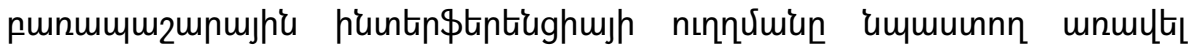

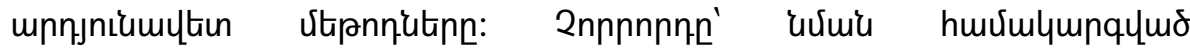

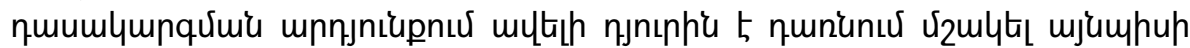

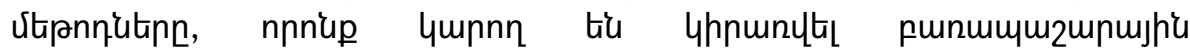

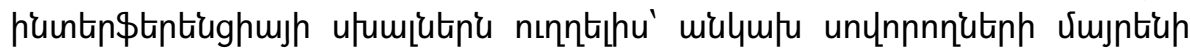
jtiquhg:

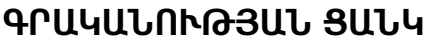

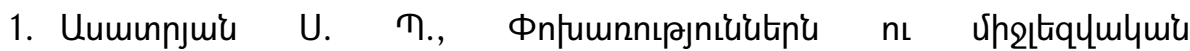

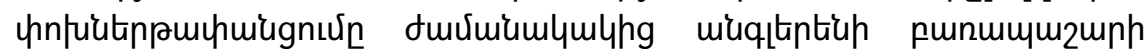

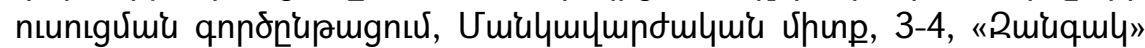

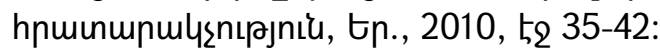

2. Алимов В. В., Интерференция в переводе. (На материале профрессионально ориентированной межкультурной коммуникации и перевода в сфере профессиональной коммуникации), дисс. доктора филологических наук, Москва, 2005, 260 стр.

3. Вященко В. С., Предупреждение и преодоление лексической интерференции в английской речи студентов II курса, дисс. кандидата педагогических наук, Ленинград, 1979, 170 стр.

4. Ценгер С. С., Морфологическая интерференция при контакте французского и русского языков, дисс. кандидата филологических наук, Москва, 2014, 233 стр.

5. Шимановская Л. А., Языковая интерференция и ее проявление в русско-английском переводе научной статьи по химической технологии, Вестник Казанского технологического университета, 17(9), 2014, c. 388-393.

6. Dweik B. S., \& Othman Z. A., Lexical and grammatical interference in the translation of written texts from Arabic into English, Academic Research International, 8(3), 2017, p. 65-70.

7. Koessler M., et Deroquigny J., Les faus amis ou les trahisons du vocabularie anglais, Conseil aux traducteurs, Paris, 1928, pp. 387. 


\section{REFERENCES}

1. Asatryan S. P., Poxarowtyownnern ow mijlezvakan poxnertapancowmy jhamanakakic anglereni barapashari owsowcman gorcyntacowm, Mankavarjhakan mitq, 3-4, «Zangak» hratarakchowtyown, Er\&an, 2010, ej 35-42.

2. Alimov V. V., Interferencija $v$ perevode. ( $\mathrm{Na}$ materiale professionalno orientirovannoj mezhkulturnoj kommunikacii $i$ perevoda $v$ sfere professionalnoj kommunikacii). Dissertacija na soiskanie uchenoj stepeni doktora filologicheskih nauk, Moskva, 2005, 260 str.

3. Vjashhenko V. S., Preduprezhdenie i preodolenie leksicheskoj interferencii $\checkmark$ anglijskoj rechi studentov II kursa, Dissertacija na soiskanie uchenoj stepen ikandidata pedagogicheskih nauk, Leningrad, 1979, 170 str.

4. Cenger S. S., Morfologicheskaja interferencija pri kontakte francuzskogo i russkogo jazykov. Dissertacija na soiskanie uchenoj stepeni kandidata filologicheskih nauk, Moskva, 2014, 233 str.

5. Shimanovskaja L. A., Jazykovaja interferencija i ee projavlenie $v$ russkoanglijskom perevode nauchnoj stat'i po himicheskoj tehnologii, Vestnik Kazanskogo tehnologicheskogo universiteta, 17(9), 2014, s. 388-393.

\section{АСМИК БИШАРЯН - ЛЕКСИЧЕСКАЯ ИНТЕРФЕРЕНЦИЯ И ЕЕ ВИДЫ В МЕТОДИКЕ ПРЕПОДАВАНИЯ АНГЛИЙСКОГО ЯЗЫКА}

Ключевые слова: лексическая интерфреренция, разновидности лексической интерференци, межъязыковая лексическая интерференция, внутриязыковая лексическая интерференция

Целью данной статьи является изучение лексической интерференции и ее видов. Приведены определения этого понятия, которые даны армянскими, российскими и западными учеными. Автор солидарен с мнением исследователей (С. Асатрян, С. Ценгер и др.), определяющих лексическую интерференцию как негативное влияние особенностей родного языка на изучаемый иностранный. В статье рассматриваются виды лексической интерференции, анализируются их характерные признаки, приводятся соответствующие примеры. Автор представляет собственную систематизированную версию классификации лексической интерференции, подчеркивая ее преимущество и целесообразность применения. Отмечается, что данная классификация позволяет разрабатывать методы, которые могут быть использованы для 
исправления ошибок, вызванных лексической интерференцией, в процессе изучения любого иностранного языка.

\section{HASMIK BISHARYAN - LEXICAL INTERFERENCE AND ITS CLASSIFICATIONS IN ENGLISH TEACHING METHODOLOGY}

Keywords: lexical interference, types of lexical interference, interlingual lexical interference, intralingual lexical interference

The purpose of the article is the study of lexical interference and its classifications. The definitions of the term proposed by Armenian, Russian and Western scholars are presented. The author is concordant with the researchers (S. Asatryan, S. Tsenger et al.) who define lexical interference as a negative transfer of the native language in the target language. The types of lexical interference are discussed according to the characteristic features of language units, parts of speech they belong to, as well as the nature and the direction of their origin. Each type is followed by relevant examples. The author presents her own systematized version of classifying lexical interference, emphasizing its advantages and appropriateness of use. It is mentioned that the particular classification contributes to the development of the methods used to correct lexical interference errors made in any language.

Utplyujugytta th' 19.03.2021

9purunuylti 5' 22.03.2021

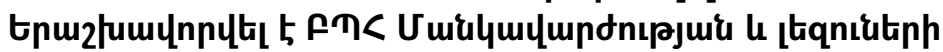

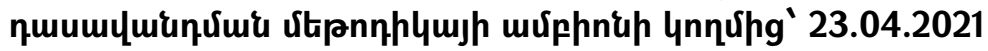

